MATHEMATICS OF COMPUTATION

Volume 79, Number 272, October 2010, Pages 2387-2394

S 0025-5718(10)02345-8

Article electronically published on April 26, 2010

\title{
THE SMALLEST PERRON NUMBERS
}

\author{
QIANG WU
}

\begin{abstract}
A Perron number is a real algebraic integer $\alpha$ of degree $d \geq 2$, whose conjugates are $\alpha_{i}$, such that $\alpha>\max _{2<i<d}\left|\alpha_{i}\right|$. In this paper we compute the smallest Perron numbers of degree $d \leq 24$ and verify that they all satisfy the Lind-Boyd conjecture. Moreover, the smallest Perron numbers of degree 17 and 23 give the smallest house for these degrees. The computations use a family of explicit auxiliary functions. These functions depend on generalizations of the integer transfinite diameter of some compact sets in $\mathbb{C}$
\end{abstract}

\section{INTRODUCTION}

Let $\boldsymbol{\alpha}$ be an algebraic integer of degree $d$, whose conjugates are $\boldsymbol{\alpha}_{1}=\boldsymbol{\alpha}$, $\boldsymbol{\alpha}_{2}, \ldots, \boldsymbol{\alpha}_{d}$ and

$$
P=X^{d}+b_{1} X^{d-1}+\cdots+b_{d-1} X+b_{d}
$$

its minimal polynomial. A Perron number, which was defined by Lind [LN1], is a real algebraic integer $\boldsymbol{\alpha}$ of degree $d \geq 2$ such that $\boldsymbol{\alpha}>\max _{2 \leq i \leq d}\left|\boldsymbol{\alpha}_{i}\right|$. Any Pisot number or Salem number is a Perron number. From the Perron-Frobenius theorem, if $A$ is a nonnegative integral matrix which is aperiodic, i.e. some power of $A$ has strictly positive entries, then its spectral radius $\boldsymbol{\alpha}$ is a Perron number. Lind has proved the converse, that is to say, if $\boldsymbol{\alpha}$ is a Perron number, then there is a nonnegative aperiodic integral matrix whose spectral radius is $\boldsymbol{\alpha}$. Lind LN2] has investigated the arithmetic of the Perron numbers. The set of Perron numbers is closed under addition and multiplication. Moreover, if $\boldsymbol{\alpha}_{1}, \boldsymbol{\alpha}_{2}, \boldsymbol{\alpha}_{3}$ are Perron numbers and $\boldsymbol{\alpha}_{3}=\boldsymbol{\alpha}_{1} \boldsymbol{\alpha}_{2}$, then $\boldsymbol{\alpha}_{1}, \boldsymbol{\alpha}_{2} \in Q\left(\boldsymbol{\alpha}_{3}\right)$. He has also shown that every Perron number can be factored into a finite product of irreducible Perron numbers, and that there are only finitely many such factorizations.

The Perron numbers and their applications were also studied by many people such as D. Boyd BO1, BO2, A. Dubickas DU1, DU2, D. Lind [LN1, LN2] and A. Schinzel $\mathrm{SC}$.

Lind LN1] conjectured that the smallest Perron number of degree $d \geq 2$ should have minimal polynomial $X^{d}-X-1$. Boyd BO1 has computed all smallest Perron numbers of degree $d \leq 12$, and Lind's conjecture turns out to be true if $d=2,3,4,6,7,8,10$, but false if $d>3$ and $d \equiv 3$ or $d \equiv 5 \bmod 6$. So in BO1, we have

Received by the editor June 9, 2009 and, in revised form, August 21, 2009.

2010 Mathematics Subject Classification. Primary 11C08, 11R06, 11 Y40.

Key words and phrases. Algebraic integer, maximal modulus, Perron numbers, explicit auxiliary functions, integer transfinite diameter.

This Project was supported by the Natural Science Foundation of Chongqing grant CSTC no. 2008BB0261. 
Conjecture (Lind-Boyd). The smallest Perron number of degree $d \geq 2$ has minimal polynomial

$$
\begin{array}{rlr}
X^{d}-X-1 \text { if } d \neq 3,5 & \bmod 6, \\
\left(X^{d+2}-X^{4}-1\right) /\left(X^{2}-X+1\right) & \text { if } d \equiv 3 & \bmod 6, \\
\left(X^{d+2}-X^{2}-1\right) /\left(X^{2}-X+1\right) \text { if } d \equiv 5 & \bmod 6 .
\end{array}
$$

For a given degree $d$, let $B>1$ be a bound sufficiently large to assure that there exists at least one Perron number $\boldsymbol{\alpha} \leq B$. In Boyd's computations, this bound was taken to be $B=(2+1 / d)^{1 / d}$ for $d \geq 3$ because $B^{d}-B-1>0$. Then let $S_{k}=\sum_{i=1}^{d} \boldsymbol{\alpha}_{i}^{k}$ for $k \geq 1$. Since all $\left|\boldsymbol{\alpha}_{i}\right| \leq \boldsymbol{\alpha} \leq B$, we have $\left|S_{k}\right| \leq d B^{k}$ for $k \geq 1$. These numbers are related to the coefficients of $P$ by Newton's relation:

$$
S_{k}+S_{k-1} b_{1}+\ldots+S_{1} b_{k-1}+k b_{k}=0
$$

for $k \geq 1$ with $b_{k}=0$ for $k>d$. The numbers $S_{k}$ also satisfy the following inequalities [BO1]:

$$
S_{2 k} \geq \frac{2}{d} S_{k}^{2}-d B^{2 k}
$$

for $k \geq 1$. The numbers $S_{k}$, with $S_{1} \leq 0$, are computed for $1 \leq i \leq d$ and this gives a large set $R_{d}$ of polynomials where $R_{d}$ denotes, as in Boyd BO1, the set of $P$ satisfying $S_{1} \leq 0,(1.1)$ and (1.2) for $k \leq d$. Let $n>d$, then $R_{n}$ denotes the set of $P$ in $R_{d}$ satisfying $b_{d} \neq 0$ and (1.1) for $k \leq n$. Then the numbers $S_{k}$ are computed by induction for $k>d$ and for each $k$ we keep only those polynomials with $\left|S_{k}\right| \leq d B^{k}$. Then for $n=3 d$ we get a reduced set $R_{3 d}$ of polynomials. By this method Boyd has, for $d=12$ with $B=1.0631 \gtrsim(2+1 / 12)^{1 / 12}$,

$$
\begin{array}{rll}
\left|R_{12}\right|=415,682,220 & \left|R_{23}\right|=37,019 & \left|R_{35}\right|=4,931 \\
\left|R_{13}\right|=23,746,503 & \left|R_{24}\right|=28,277 & \left|R_{36}\right|=4,435
\end{array}
$$

for only 30 irreducible polynomials. The last step is to keep only irreducible polynomials which are not cyclotomic and we get all algebraic integers of degree $d$ with $\max _{1 \leq i \leq d}|\boldsymbol{\alpha}| \leq B$ and so we have the smallest Perron number.

In this paper we compute all smallest Perron numbers of degree $d \leq 24$. We follow Boyd's strategy but we give better bounds for the numbers $S_{k}$ and more efficient relations between $S_{k}$ and $S_{2 k}$ than (1.2) with a family of explicit auxiliary functions. These functions are related to a generalization of the integer transfinite diameter. This hugely speeds up the search. For example, we have, for $d=12$,

$$
\begin{array}{lll}
\left|R_{12}\right|=950,484 & \left|R_{23}\right|=7,367 & \left|R_{35}\right|=1,892 \\
\left|R_{13}\right|=211,761 & \left|R_{24}\right|=6,861 & \left|R_{36}\right|=1,679 .
\end{array}
$$

The computing time for $d=12$ is a few seconds on a $2.8 \mathrm{Ghz}$ PC.

We denote the house of $\boldsymbol{\alpha}$ by

$$
\boldsymbol{\alpha}\left|=\max _{1 \leq i \leq d}\right| \boldsymbol{\alpha}_{i} \mid .
$$

Remark. In [RW], G. Rhin and the author have computed all the algebraic integers with smallest house of degree $\leq 28$. Since here, we follow the same strategy, but with greater bounds $B$, we can use the same list of polynomials for the auxiliary functions for the bounds of $\left|S_{k}\right|$ to find the smallest Perron number. But for degree 22 , the computing time becomes 50 hours and for degree 23 it will be more than 
TABLe 1. List of all Perron numbers $\boldsymbol{\alpha}$ of degree $13 \leq d \leq 24$ with $\boldsymbol{\alpha} \leq(2+1 / d)^{1 / d}$ and their minimal polynomials.

\begin{tabular}{|c|c|c|c|}
\hline$d$ & $\boldsymbol{\alpha}$ & $(2+1 / d)^{1 / d}$ & polynomial $P$ \\
\hline 13 & $1.057050 \ldots$ & $1.057832 \ldots$ & $X^{13}-X-1$ \\
\hline 14 & $1.052710 \ldots$ & $1.053393 \ldots$ & $X^{14}-X-1$ \\
\hline 15 & $1.047595 \ldots$ & $1.049585 \ldots$ & $\left(X^{17}-X^{4}-1\right) /\left(X^{2}-X+1\right)$ \\
\hline & $1.048984 \ldots$ & & $X^{15}-X-1$ \\
\hline 16 & $1.045751 \ldots$ & $1.046284 \ldots$ & $X^{16}-X-1$ \\
\hline 17 & $1.039302 \ldots$ & $1.043393 \ldots$ & $\left(X^{19}-X^{2}-1\right) /\left(X^{2}-X+1\right)$ \\
\hline & $1.042917 \ldots$ & & $X^{17}-X-1$ \\
\hline 18 & $1.040414 \ldots$ & $1.040842 \ldots$ & $X^{18}-X-1$ \\
\hline 19 & $1.038188 \ldots$ & $1.038573 \ldots$ & $X^{19}-X-1$ \\
\hline 20 & $1.036193 \ldots$ & $1.036543 \ldots$ & $X^{20}-X-1$ \\
\hline 21 & $1.033665 \ldots$ & $1.034716 \ldots$ & $\left(X^{23}-X^{4}-1\right) /\left(X^{2}-X+1\right)$ \\
\hline & $1.034397 \ldots$ & & $X^{21}-X-1$ \\
\hline 22 & $1.032770 \ldots$ & $1.033063 \ldots$ & $X^{22}-X-1$ \\
\hline 23 & $1.029320 \ldots$ & $1.031559 \ldots$ & $\left(X^{25}-X^{2}-1\right) /\left(X^{2}-X+1\right)$ \\
\hline & $1.031291 \ldots$ & & $X^{23}-X-1$ \\
\hline 24 & $1.029939 \ldots$ & $1.030186 \ldots$ & $X^{24}-X-1$ \\
\hline
\end{tabular}

800 hours of CPU time. For degree 24, the computing time would certainly be too large. So we need to improve the bounds for $\left|S_{k}\right|$ by using better auxiliary functions. Using the improvement of our algorithm given by V. Flammang [FL, we get many new polynomials $Q_{j}$ in the auxiliary function of greater degree as we explain in Section 2c and decrease the computing time for degree 23 down to 20 hours.

We give in Table 1 all Perron numbers of degree $13 \leq d \leq 24$ with $\boldsymbol{\alpha} \leq(2+$ $1 / d)^{1 / d}$ and their minimal polynomials, and we verify that all the smallest Perron numbers of degree $13 \leq d \leq 24$ satisfy the Lind-Boyd conjecture. We also get all algebraic integers $\boldsymbol{\alpha}$ of degree $d \leq 24$, which are not a root of unity, with $|\boldsymbol{\alpha}| \leq(2+1 / d)^{1 / d}$. The complete list may be obtained on request to the author (for $d=24$ see Table 4 in Section 3). The computing time, for example, for $d=24$, is 358 hours on a $2.8 \mathrm{Ghz}$ PC.

We also had in RW], for $d=29,(d \equiv 5 \bmod 6)$, a small Perron number whose minimal polynomial is

$$
\frac{X^{31}-X^{2}-1}{X^{2}-X-1} .
$$

We expect that this provides an algebraic integer $\boldsymbol{\alpha}$ of degree 29 of smallest house $(\mid \boldsymbol{\alpha}=1.023383 \ldots)$. Then it would satisfy the Lind-Boyd conjecture. So we have the following

Conjecture. The smallest Perron number of degree $d \geq 17, d \equiv 5 \bmod 6$ and $d$ is a prime number, gives the smallest house.

In Section 2, we explain how to use explicit auxiliary functions to give bounds for $S_{k}$, and relations between $S_{k}$ and $S_{2 k}$. We explain the relations between explicit auxiliary functions and integer transfinite diameter. Section 3 is devoted to the final computation to get the smallest Perron numbers of degree $d \leq 24$. 
TABLE 2

\begin{tabular}{|c|c|c|c|c|c|c|c|c|c|c|c|c|c|c|c|c|}
\hline$k$ & 1 & 2 & 3 & 4 & 5 & 6 & 7 & 8 & 9 & 13 & 17 & 23 & 33 & 48 & 60 & 72 \\
\hline$B N_{k}$ & 3 & 5 & 6 & 8 & 9 & 10 & 12 & 14 & 15 & 21 & 28 & 37 & 60 & 97 & 140 & 203 \\
\hline$B C_{k}$ & 24 & 25 & 26 & 27 & 27 & 28 & 29 & 30 & 31 & 35 & 39 & 47 & 64 & 100 & 142 & 204 \\
\hline
\end{tabular}

\section{The Bounds For $S_{k}$ With the EXPlicit AUXiLiary FUnCtions}

a. The explicit auxiliary functions for the bounds for $S_{k}$.

Compared with Boyd's strategy, the main improvement of the calculation is to compute bounds of $S_{k}$ which replace the classical bounds $\left|S_{k}\right| \leq d(2+1 / d)^{k / d}$ for $1 \leq k \leq 3 d$ by using a family of explicit auxiliary functions. For small $k$, this method improves drastically the classical bounds. In Table 2 we give an example of the two kinds of bounds for some values of $k$ for degree $d=24$. $B N_{k}$ denotes the new bound of $S_{k}$ and $B C_{k}$ is the classical one. We define the explicit auxiliary function $f$ by the formula

$$
f(z)=-\operatorname{Re}(z)-\sum_{1 \leq j \leq J} e_{j} \log \left|Q_{j}(z)\right|
$$

where $z$ is a complex number, the numbers $e_{j}$ are positive real numbers and the polynomials $Q_{j}$ are nonzero elements of $\mathbb{Z}[X]$. The numbers $e_{j}$ are always chosen to get the best auxiliary function. We denote by $m$ the minimum of $f(z)$ for $|z| \leq B$. Since the function $f$ is harmonic in this disk outside the union of small disks around the roots of the polynomials $Q_{j}$, this minimum is taken on $|z|=B$.

We have

$$
\sum_{1 \leq i \leq d} f\left(\boldsymbol{\alpha}_{i}\right) \geq m d
$$

and

$$
-S_{1} \geq d m+\sum_{1 \leq j \leq J} e_{j} \log \left|\prod_{1 \leq i \leq d} Q_{j}\left(\boldsymbol{\alpha}_{i}\right)\right| .
$$

$\prod_{1 \leq i \leq d} Q_{j}\left(\boldsymbol{\alpha}_{i}\right)$ is equal to the resultant of $P$ and $Q_{j}$. If we assume now that the polynomial $P$ does not divide any polynomial $Q_{j}$, then this is a nonzero integer. Therefore,

$$
S_{1} \leq-d m
$$

We give, for example, in Table 3 the list of polynomials $Q_{j}$ and the numbers $e_{j}$ which are used in the auxiliary function for $S_{1}$ for $d=24$. With this auxiliary function, we have $-S_{1} \geq-3.85392590$, i.e. $S_{1} \leq 3$ as in Table 2 .

By symmetry, the same inequality is valid for $-S_{1}$. If we replace $B$ by $B^{k}$ and the numbers $\boldsymbol{\alpha}_{i}$ by the numbers $\pm \boldsymbol{\alpha}_{i}{ }^{k}$ we get upper bounds for $\pm S_{k}$.

b. The explicit auxiliary functions which give relations between $S_{k}$ and $S_{2 k}$.

In Boyd [BO1, we have the classical relation (1.2) between $S_{k}$ and $S_{2 k}$. Here we exploit the relations between $S_{k}$ and $S_{2 k}$ that will be given by explicit auxiliary functions of the following type:

$$
f(z)=\operatorname{Re}\left(z^{2}\right)-e_{0} \operatorname{Re}(z)-\sum_{1 \leq j \leq J} e_{j} \log \left|Q_{j}(z)\right|
$$


TABLE 3. List of $Q_{j}$ and $e_{j}$ in the auxiliary function for $S_{1}$ for $d=24 . d_{j}=\operatorname{deg} Q_{j}$, and the coefficient of $Q_{j}$ are written from degree 0 to $d_{j}$.

\begin{tabular}{|c|c|c|c|c|c|c|c|c|c|c|c|c|c|c|c|}
\hline$e_{j}$ & $d_{j}$ & & & & $\mathrm{Oe}$ & ents & of 6 & & & & & & & & \\
\hline 0.60641770 & 1 & -1 & 1 & & & & & & & & & & & & \\
\hline 0.27754798 & 2 & 1 & -1 & 1 & & & & & & & & & & & \\
\hline 0.05030884 & 2 & 1 & 0 & 1 & & & & & & & & & & & \\
\hline 0.10381486 & 4 & 1 & -1 & 1 & -1 & 1 & & & & & & & & & \\
\hline 0.00590394 & 4 & 1 & 0 & 0 & 0 & 1 & & & & & & & & & \\
\hline 0.01086250 & 4 & 1 & 0 & -1 & 0 & 1 & & & & & & & & & \\
\hline 0.04953738 & 6 & 1 & -1 & 1 & -1 & 1 & -1 & 1 & & & & & & & \\
\hline 0.00749535 & 6 & 1 & 0 & 0 & -1 & 0 & 0 & 1 & & & & & & & \\
\hline 0.00661499 & 6 & 1 & 0 & 0 & 1 & 0 & 0 & 1 & & & & & & & \\
\hline 0.01545423 & 8 & 1 & -1 & 0 & 1 & -1 & 1 & 0 & -1 & 1 & & & & & \\
\hline 0.00246685 & 8 & 2 & -4 & 4 & -2 & 1 & -1 & 2 & -2 & 1 & & & & & \\
\hline 0.01273706 & 9 & 1 & -1 & 1 & -1 & 1 & 0 & -1 & 2 & -2 & 1 & & & & \\
\hline 0.00767717 & 10 & 1 & -2 & 3 & -3 & 2 & -1 & 2 & -3 & 3 & -2 & 1 & & & \\
\hline 0.00052989 & 10 & 1 & -1 & 1 & -1 & 1 & -1 & 2 & -3 & 3 & -2 & 1 & & & \\
\hline 0.00102170 & 10 & 2 & -4 & 5 & -4 & 2 & -1 & 2 & -3 & 3 & -2 & 1 & & & \\
\hline 0.00481569 & 12 & 1 & -2 & 2 & -1 & 1 & -1 & 1 & -1 & 1 & -1 & 2 & -2 & & 1 \\
\hline 0.00148998 & 14 & $\begin{array}{r}3 \\
-3\end{array}$ & $\begin{array}{r}-10 \\
1\end{array}$ & 19 & -25 & 25 & -19 & 12 & -7 & 5 & -5 & 6 & -6 & & 5 \\
\hline 0.00161833 & 14 & $\begin{array}{r}2 \\
-3\end{array}$ & $\begin{array}{r}-5 \\
1\end{array}$ & 7 & -7 & 7 & -7 & 7 & -6 & 5 & -5 & 6 & -6 & & 5 \\
\hline 0.00014458 & 15 & $\begin{array}{l}1 \\
2\end{array}$ & $\begin{array}{l}-1 \\
-2\end{array}$ & $\begin{array}{l}1 \\
1\end{array}$ & -3 & 6 & -8 & 10 & -10 & 8 & -6 & 4 & -1 & -1 & 1 \\
\hline 0.00014745 & 16 & $\begin{array}{l}2 \\
-5\end{array}$ & $\begin{array}{r}-6 \\
5\end{array}$ & $\begin{array}{l}11 \\
-3\end{array}$ & $\begin{array}{r}-14 \\
1\end{array}$ & 13 & -8 & 2 & 4 & -8 & 9 & -7 & 3 & & 2 \\
\hline 0.00063289 & 16 & $\begin{array}{c}4 \\
-25\end{array}$ & $\begin{array}{r}-17 \\
17\end{array}$ & $\begin{array}{l}39 \\
-8\end{array}$ & $\begin{array}{r}-63 \\
2\end{array}$ & 83 & -95 & 98 & -92 & 79 & -62 & 47 & -37 & 31 & 1 \\
\hline 0.00095988 & 20 & $\begin{array}{r}-3 \\
199\end{array}$ & $\begin{array}{c}12 \\
-117\end{array}$ & $\begin{array}{r}-27 \\
47\end{array}$ & $\begin{array}{r}45 \\
-4\end{array}$ & $\begin{array}{c}-66 \\
-12\end{array}$ & $\begin{array}{c}93 \\
11\end{array}$ & $\begin{array}{c}-132 \\
-5\end{array}$ & $\begin{array}{c}184 \\
1\end{array}$ & -242 & 292 & -320 & 314 & -271 & \\
\hline 0.00477448 & 22 & $\begin{array}{c}3 \\
-16\end{array}$ & $\begin{array}{r}-12 \\
18\end{array}$ & $\begin{array}{l}23 \\
-13\end{array}$ & $\begin{array}{r}-24 \\
8\end{array}$ & $\begin{array}{r}7 \\
-7\end{array}$ & $\begin{array}{r}25 \\
9\end{array}$ & $\begin{array}{c}-58 \\
-10\end{array}$ & $\begin{array}{r}78 \\
8\end{array}$ & $\begin{array}{l}-79 \\
-4\end{array}$ & $\begin{array}{r}65 \\
1\end{array}$ & -42 & 17 & & 4 \\
\hline
\end{tabular}

where the numbers $e_{j}$ and the polynomials $Q_{j}$ are as in paragraph a. If $m$ is the minimum of $f(z)$ for $|z| \leq B$, by the same argument as in paragraph a, we get

$$
S_{2}-e_{0} S_{1} \geq m d \text {. }
$$

If we assume that $S_{1}$ has the value $\boldsymbol{\sigma}$, then $S_{2} \geq d m+e_{0} \boldsymbol{\sigma}$. We optimize the numbers $e_{0}, \ldots, e_{J}$ to get a maximal value of $d m+e_{0} \boldsymbol{\sigma}$. Therefore, we get a lower bound for $S_{2}$ depending on the value of $\boldsymbol{\sigma}$. This gives a better bound than the one which was given in paragraph a if we take $\boldsymbol{\sigma}$ close to its upper bound. If we replace in $(2.3), e_{0} \operatorname{Re}(z)$ by $-e_{0} \operatorname{Re}(z)$, we get the same lower bound for $S_{2}$ when $S_{1}$ has the value $-\boldsymbol{\sigma}$. We may also replace $\operatorname{Re}\left(z^{2}\right)$ by $-\operatorname{Re}\left(z^{2}\right)$ and get upper bounds for $S_{2}$. Then replacing $B$ by $B^{k}$ we get bounds for $S_{2 k}$ when $S_{k}$ has values which are close to its bounds. 
c. Relations between explicit auxiliary functions and integer transfinite diameter.

If, inside the auxiliary function of (2.1), we replace the real numbers $e_{j}$ by rational numbers, we may write

$$
f(z)=-\operatorname{Re}(z)-\frac{t}{h} \log |H(z)|
$$

where $H$ is in $\mathbb{Z}[X]$ of degree $h$ and $t$ is a positive real number. We want to get a function $f$ whose minimum $m$ in $|z| \leq B$ is as large as possible. That is to say that we seek a polynomial $H \in \mathbb{Z}[X]$ such that

$$
\sup _{|z| \leq B}|H(z)|^{t / h} e^{\operatorname{Re}(z)} \leq e^{-m}
$$

Now, if we suppose that $t$ is fixed, say $t=1$, it is clear that we need to get an effective upper bound for the quantity

$$
t_{\mathbb{Z}, \varphi}(|z| \leq B)=\liminf _{\substack{h \geq 1 \\ h \rightarrow \infty}} \inf _{\substack{H \in \mathbb{Z}[X] \\ \operatorname{deg} H=h}} \sup _{|z| \leq B}|H(z)|^{t / h} \varphi(z)
$$

in which we use the weight $\varphi(z)=e^{\operatorname{Re}(z)}$. To get an upper bound for $t_{\mathbb{Z}, \boldsymbol{\varphi}}(|z| \leq B)$, it is sufficient to get an explicit polynomial $H \in \mathbb{Z}[X]$ and then to use the sequence of the successive powers of $H$.

This is a generalization of the integer transfinite diameter. For any $h \geq 1$ we say that a polynomial $H$ (not always unique) is an integer Chebyshev polynomial if the quantity $\sup _{|z| \leq B}|H(z)|^{t / h} \boldsymbol{\varphi}(z)$ is minimum. With the author's algorithm [WU], we compute polynomials $H$ of degree less than 30 and take their irreducible factors as polynomials $Q_{j}$. For example, for the bound of $\left|S_{1}\right|$ of smallest Perron number of degree 24, we start with the polynomials $X-1$ and $X^{2}-X+1$. With the semi-infinite linear programming method that was introduced into number theory by C. J. Smyth $\left[\mathrm{SM}\right.$, we get the best $e_{1}$ and $e_{2}$. We then have $f_{1}(z)=-\operatorname{Re}(z)-$ $e_{1} \log |z-1|-e_{2} \log \left|z^{2}-z+1\right|$. We deduce the value of $t_{1}=e_{1} \operatorname{deg}(X-1)+$ $e_{2} \operatorname{deg}\left(X^{2}-X+1\right)$. Now, we search for a polynomial $Q$ of fixed degree $d$ (say 25), such that $H(z)=(z-1)^{\left[d e_{1} / t_{1}\right]}\left(z^{2}-z+1\right)^{\left[d e_{2} / t_{1}\right]} Q(z)$ is small on $|z| \leq B$. We take a finite set of points $z_{i}$ in $|z| \leq B$ containing all the local minima of $f_{1}(z)$. LLL will give polynomials $Q_{j}$ such that all $H\left(z_{i}\right)$ are small. We optimize the function

$$
f_{2}(z)=-\operatorname{Re}(z)-e_{1} \log |z-1|-e_{2} \log \left|z^{2}-z+1\right|-\sum_{j} e_{j} \log \left|Q_{j}\right|
$$

We keep only $Q_{j}$ when $e_{j} \neq 0$. Then we have a new bound of $\left|S_{1}\right|$ which is better than the previous one. We repeat LLL and optimization of the auxiliary function and finally we have the function in Table 2. More details can be found in FRSE, RW] and FL.

With this method, for $d=24$, we find 143 polynomials $Q_{j}$ for the family of explicit auxiliary functions for $S_{k}$ for all $1 \leq k \leq 72$, and the largest degree of the polynomials $Q_{j}$ is 22 . We have used 125 polynomials for the relations (2.3) and 94 polynomials if we replace $e_{0} \operatorname{Re}(z)$ by $-e_{0} \operatorname{Re}(z)$. So we have a set of 341 different polynomials, and this set contains 35 of the 42 polynomials used in [RW]. 
TABLE 4. List of the minimal noncyclotomic polynomials of algebraic integers of degree 24 with small house.

\begin{tabular}{|c|c|}
\hline house of $P$ & polynomial $P$ \\
\hline $1.017730 \ldots$ & $X^{24}+X^{16}-1$ \\
\hline $1.022651 \ldots$ & $X^{24}-X^{20}+X^{12}-X^{8}+1$ \\
\hline $1.024177 \ldots$ & $X^{24}+X^{8}-1$ \\
\hline $1.024596 \ldots$ & $X^{24}+X^{21}+X^{12}-X^{6}+1$ \\
\hline $1.025758 \ldots$ & $\left(X^{28}+X^{2}+1\right) /\left(\boldsymbol{\Phi}_{3} \boldsymbol{\Phi}_{6}\right)$ \\
\hline $1.027157 \ldots$ & $\left(X^{30}+X^{15}-X^{3}+1\right) /\left(\boldsymbol{\Phi}_{4} \boldsymbol{\Phi}_{12}\right)$ \\
\hline $1.027482 \ldots$ & $\left(X^{26}-X+1\right) / \Phi_{6}$ \\
\hline $1.028309 \ldots$ & $X^{24}+X^{20}+X^{16}+1$ \\
\hline $1.028513 \ldots$ & $X^{24}-X^{6}+1$ \\
\hline $1.029302 \ldots$ & $X^{24}-X^{12}+2$ \\
\hline $1.029312 \ldots$ & $X^{24}+X^{21}+X^{18}+X^{15}+1$ \\
\hline $1.029354 \ldots$ & $\left(X^{27}-X^{14}+X-1\right) /\left(\boldsymbol{\Phi}_{1} \boldsymbol{\Phi}_{4}\right)$ \\
\hline $1.029841 \ldots$ & $X^{24}+X+1$ \\
\hline $1.029939 \ldots$ & $X^{24}-X-1$ Perron \\
\hline $1.030138 \ldots$ & $X^{24}-X^{4}+1$ \\
\hline
\end{tabular}

\section{The FinAl COMPUTATION}

For all algebraic integers with $|\boldsymbol{\alpha}| \leq(2+1 / d)^{1 / d}$, we have the bounds of $S_{k}$ for $k$ from 1 to $3 d$. Now we follow Boyd's strategy BO1] to compute the coefficients of the polynomial $P$ with $b_{1} \geq 0$.

As in Section 1, for $d=24$, we have $\left|R_{24}\right|=12,132,432,709,704$ and $\left|R_{72}\right|=$ 33,132 . Moreover, we refine some steps as follows in our computation:

We take $\left|b_{d}\right|= \pm 1, \pm 2$, because $b_{d} \in \mathbb{Z}$ and

$$
\left|b_{d}\right|=\prod_{j=1}^{d}\left|\boldsymbol{\alpha}_{j}\right| \leq B^{d} \leq 2+\frac{1}{d} .
$$

Moreover, by Corollary 2 of Matveev [MAT], we know that, for any algebraic integer $\boldsymbol{\alpha}$ of degree $d$, if

$$
|\boldsymbol{\alpha}| \leq \exp \left(\frac{\ln \left(d+\frac{1}{2}\right)}{d^{2}}\right)
$$

then $\boldsymbol{\alpha}$ is a root of unity. So we eliminate, by the Schur-Cohn algorithm [MAR, any polynomial $P$ of $R_{3 d}$ which satisfies the condition (3.1). We obtain 48 polynomials for $d=24$. Then we use Pari $[\mathrm{PA}$ to eliminate any polynomial with a cyclotomic factor, get all the irreducible polynomials, and compute explicitly their roots.

Then we find all irreducible polynomials of degree $d$ which satisfy $|\boldsymbol{\alpha}| \leq(2+$ $1 / d)^{1 / d}$, so we have all smallest Perron numbers for $d \leq 24$, and also all algebraic integers $\boldsymbol{\alpha}$ with small house $\left(\leq(2+1 / d)^{1 / d}\right)$. We list in Table 4 all the minimal polynomials of degree 24 of algebraic integers which are not a root of unity with small house that we get by our computations. We give only one polynomial if there exist several polynomials which have the same house. Here $\boldsymbol{\Phi}_{1}=X-1$, $\boldsymbol{\Phi}_{3}=X^{2}+X+1, \boldsymbol{\Phi}_{4}=X^{2}+1, \boldsymbol{\Phi}_{6}=X^{2}-X+1$ and $\boldsymbol{\Phi}_{12}=X^{4}-X^{2}+1$ are cyclotomic polynomials. 
As in WU, Section 2, when we use the auxiliary functions, we suppose that the polynomial $P$ does not divide any polynomial $Q_{j}\left( \pm X^{k}\right)$ for $k \leq 3 d$ (i.e. $\pm \boldsymbol{\alpha}_{i}^{k}$ is not a zero of $Q_{j}$ for all $j$ and $k$ ). So it is necessary to add to the list of Table 4 the nonprimitive polynomials $P$ obtained as polynomials $Q_{j}\left( \pm X^{k}\right)$ whose house is less than $(2+1 / d)^{1 / d}$ where $d=k \operatorname{deg} Q_{j}$. For example, if $Q_{j}=X^{2}-2 X+2$, then all the polynomials $X^{2 k}-2 X^{k}+2$ whose house $\leq(2+1 / 2 k)^{1 / 2 k}$ have to be added to the list.

\section{ACKNowledgement}

This work would not have been possible without the precious help of Professor Georges Rhin, I am very much indebted to him.

\section{REFERENCES}

[BO1] D. W. Boyd, The maximal modulus of an algebraic integer, Math. Comp. 45 (1985), 243-249. MR790657 (87c:11097)

[BO2] D. W. Boyd, Perron units which are not Mahler measures, Ergodic Theory Dynam. Systems 6 (1986), 485-488. MR873427 (88e:11100)

[DU1] A. Dubickas, Some Diophantine properties of the Mahler measure, Math. Notes 72 (2002), 763-767. MR 1964142 (2004b:11148)

[DU2] A. Dubickas, On numbers which are Mahler measures, Monatsh. Math. 141 (2004), 119126. MR2037988 (2005a:11164)

[FL] V. Flammang, Trace of totally positive algebraic integers and integer transfinite diameter, Math. Comp. 78 (2009), 1119-1125. MR2476574

[FRSE] V. Flammang, G. Rhin and J.M. Sac-Épée, Integer transfinite diameter and polynomials of small Mahler measure, Math. Comp. 75 (2006), 1527-1540. MR2219043(2007d:11138)

[LN1] D. Lind, Entropies and factorizations of topological Markov shifts, Bull. Amer. Math. Soc. (N.S.) 9 (1983), 219-222. MR707961 (84j:54010)

[LN2] D. Lind, The entropies of topological Markov shifts and a related class of algebraic integers, Ergodic Theory Dynamical Systems 4 (1984), 283-300. MR766106 (86c:58092)

[MAR] M. Marden, Geometry of polynomials, Second edition. Mathematical Surveys, no. 3, Amer. Math. Soc., Providence, Rhode Island (1966). MRMR0225972 (37:1562)

[MAT] E.M. Matveev, On the size of algebraic integers, Math. Notes 49 (1991), 437-438. MR:1119233 (92g:11070)

[PA] C. Batut, K. Belabas, D. Bernardi, H. Cohen, M. Olivier, GP-Pari version 2.1.6, 2002.

[RW] G. Rhin, Q. Wu, On the smallest value of the maximal modulus of an algebraic integer, Math. Comp. 76 (2007), 1025-1038. MR2291848 (2007m:11146)

[SC] A. Schinzel, A class of algebraic numbers, Tatra Mt. Math. Publ. 11 (1997), 35-42. MR 1475503 (98i:11089)

[SM] C. J. Smyth, The mean values of totally real algebraic integers, Math. Comp. 42 (1984), 663-681. MR.736460 (86e:11115)

[WU] Q. Wu, On the linear independence measure of logarithms of rational numbers, Math. Comp. 72 (2003), 901-911. MR1954974(2003m:11111)

Department of Mathematics, Southwest University of China, 2 Tiansheng Road Beibei, 400715 Chongqing, China

E-mail address: qiangwu@swu.edu.cn 